\title{
An Experimental Review on Elliptic Flow of Strange and Multistrange Hadrons in Relativistic Heavy Ion Collisions
}

\author{
Shusu Shi \\ Key Laboratory of Quarks and Lepton Physics (MOE) and Institute of Particle Physics, Central China Normal University, \\ Wuhan 430079, China \\ Correspondence should be addressed to Shusu Shi; shiss@mail.ccnu.edu.cn
}

Received 1 June 2016; Accepted 10 August 2016

Academic Editor: Xiaochun He

Copyright (C) 2016 Shusu Shi. This is an open access article distributed under the Creative Commons Attribution License, which permits unrestricted use, distribution, and reproduction in any medium, provided the original work is properly cited. The publication of this article was funded by $\mathrm{SCOAP}^{3}$.

Strange hadrons, especially multistrange hadrons, are good probes for the early partonic stage of heavy ion collisions due to their small hadronic cross sections. In this paper, I give a brief review on the elliptic flow measurements of strange and multistrange hadrons in relativistic heavy ion collisions at Relativistic Heavy Ion Collider (RHIC) and Large Hadron Collider (LHC).

\section{Introduction}

At the early stage of high energy relativistic heavy ion collisions, a hot and dense, strongly interacting medium named Quark Gluon Plasma (QGP) is created $[1,2]$. The subsequent system evolution is determined by the nature of the medium. Experimentally, the dynamics of the system evolution has been studied by measuring the azimuthal anisotropy of the particle production relative to the reaction plane [3-5]. The centrality of the collision, defined by the transverse distance between the centers of the colliding nuclei called the impact parameter, results in an "almond-shaped" overlap region that is spatially azimuthal anisotropic. It is generally assumed that the initial spatial anisotropy in the system is converted into momentum-space anisotropy through rescatterings [6]. The elliptic flow, $v_{2}$, which is the second Fourier coefficient of the azimuthal distribution of produced particles with respect to the reaction plane, is defined as $v_{2}=\langle\cos 2(\varphi-\Psi)\rangle$, where $\varphi$ is the azimuthal angle of produced particle and $\Psi$ is the azimuthal angle of the reaction plane. The initial anisotropy in the coordinate space diminishes rapidly as the system expands. Thus, the driving force of $v_{2}$ quenches itself. Due to the self-quenching effect, the elliptic flow provides information about the dynamics at the early stage of the collisions [79]. Elliptic flow can provide information about the pressure gradients, the effective degrees of freedom, the degree of thermalization, and equation of state of the matter created at the early stage [5]. However, early dynamic information might be obscured by later hadronic rescatterings $[10,11]$. Strange hadrons, especially multistrange hadrons, and the $\phi$ meson are believed to be less sensitive to hadronic rescatterings in the late stage of collisions, as their freeze-out temperatures are close to the phase transition temperature and their hadronic interaction cross sections are expected to be small $[12,13]$. In this paper, I am going to review the elliptic flow results of strange and multistrange hadron in relativistic heavy ion collisions from RHIC to LHC energies.

\section{Discussions}

2.1. Centrality and System Size Dependence. The values of $v_{2}$ are usually divided by the initial spatial anisotropy, eccentricity, to remove the geometric effect in order to study the centrality and system size dependence of $v_{2}$. The participant eccentricity is the initial configuration space eccentricity of the participants which is defined by $[14,15]$

$$
\varepsilon_{\mathrm{part}}=\frac{\sqrt{\left(\sigma_{y}^{2}-\sigma_{x}^{2}\right)+4\left(\sigma_{x y}^{2}\right)}}{\sigma_{y}^{2}+\sigma_{x}^{2}},
$$

where $\sigma_{x}^{2}=\left\langle x^{2}\right\rangle-\langle x\rangle^{2}, \sigma_{y}^{2}=\left\langle y^{2}\right\rangle-\langle y\rangle^{2}$, and $\sigma_{x y}=\langle x y\rangle-$ $\langle x\rangle\langle y\rangle$, with $x, y$ being the position of the participating 

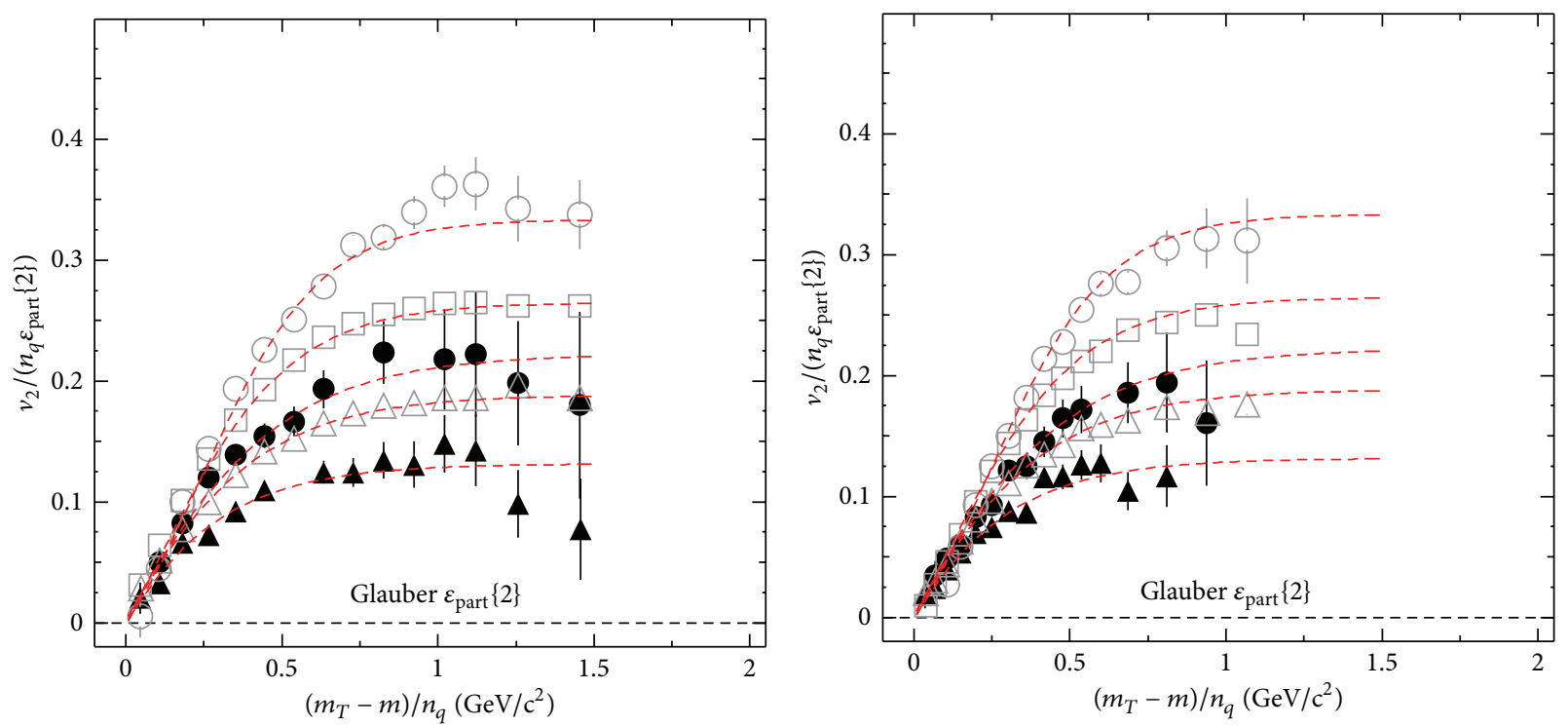

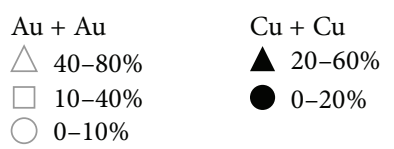

(a) $K_{S}^{0}$

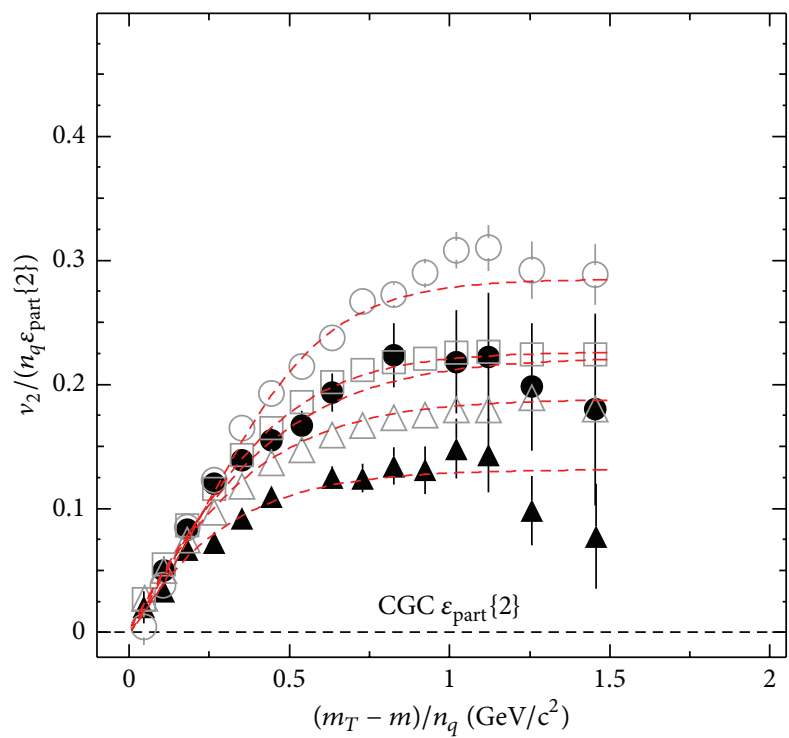

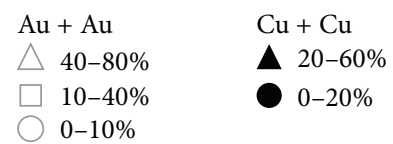

(b) $\Lambda$

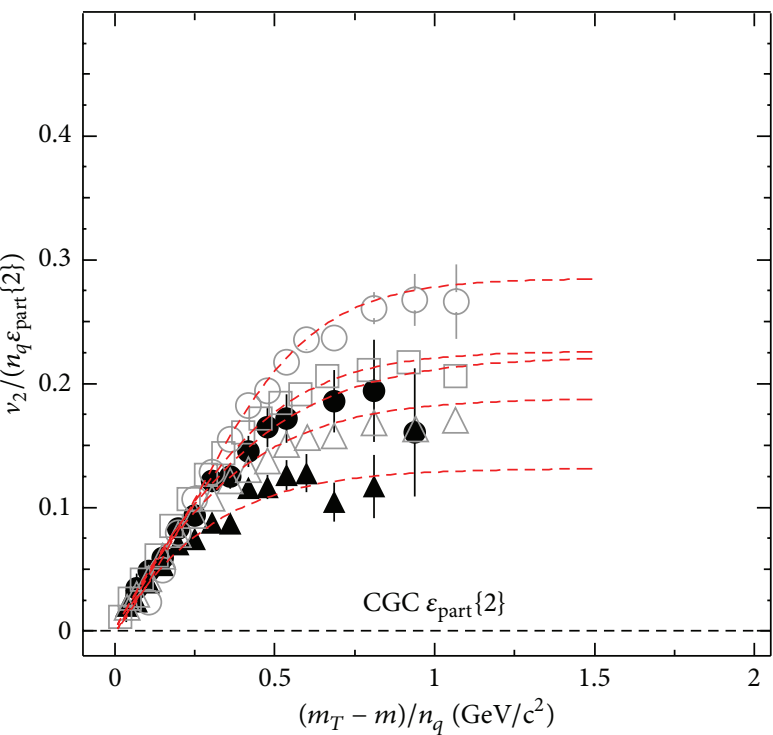

(c) $K_{S}^{0}$
$\mathrm{Au}+\mathrm{Au}$
$\mathrm{Cu}+\mathrm{Cu}$
$\triangle 40-80 \%$
A $20-60 \%$
$\mathrm{Cu}+\mathrm{Cu}$
$\square \quad 10-40 \%$
- $0-20 \%$
$\triangle 40-80 \%$
- 20-60\%
0-10\%
$10-40 \%$

(d) $\Lambda$

FIGURE 1: Centrality dependence of $v_{2}$ scaled by number of quarks and participant eccentricity $\left(v_{2} /\left(n_{q} \varepsilon_{\text {part }}\{2\}\right)\right)$ for $K_{S}^{0}$ and $\Lambda$ as a function of $\left(m_{T}-m\right) / n_{q}$ in $0-10 \%, 10-40 \%$, and $40-80 \% \mathrm{Au}+$ Au collisions and $0-20 \%$ and $20-60 \% \mathrm{Cu}+\mathrm{Cu}$ collisions at $\sqrt{s_{N N}}=200 \mathrm{GeV}[14]$.

nucleons in the transverse plane. The root mean square of the participant eccentricity,

$$
\varepsilon_{\text {part }}\{2\}=\sqrt{\left\langle\varepsilon_{\text {part }}^{2}\right\rangle},
$$

is calculated from the Monte Carlo Glauber model [16] and Color Glass Condensate (CGC) model [17].

Figure 1 shows the centrality and system size dependence of $K_{S}^{0}$ and $\Lambda v_{2}$ in $\sqrt{s_{N N}}=200 \mathrm{GeV}$ heavy ion collisions [14]. The eccentricity scaled $v_{2}$ has been further normalized 


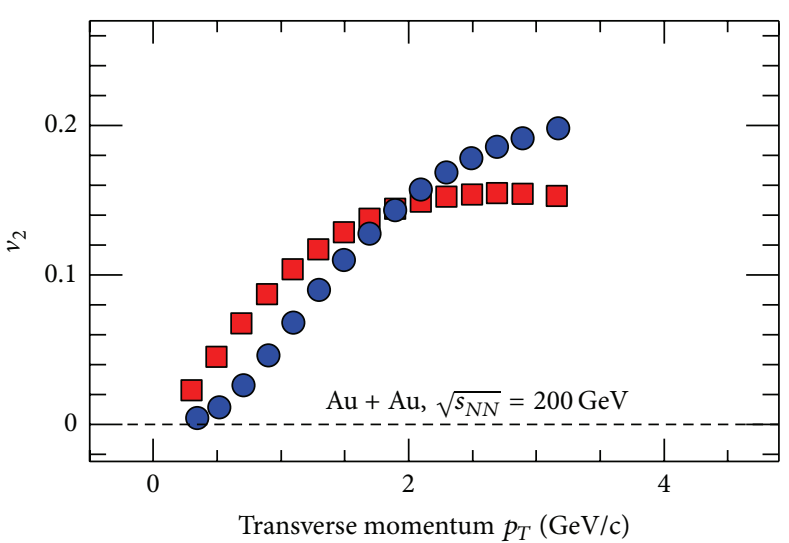

$\bigcirc$
$\square \pi$

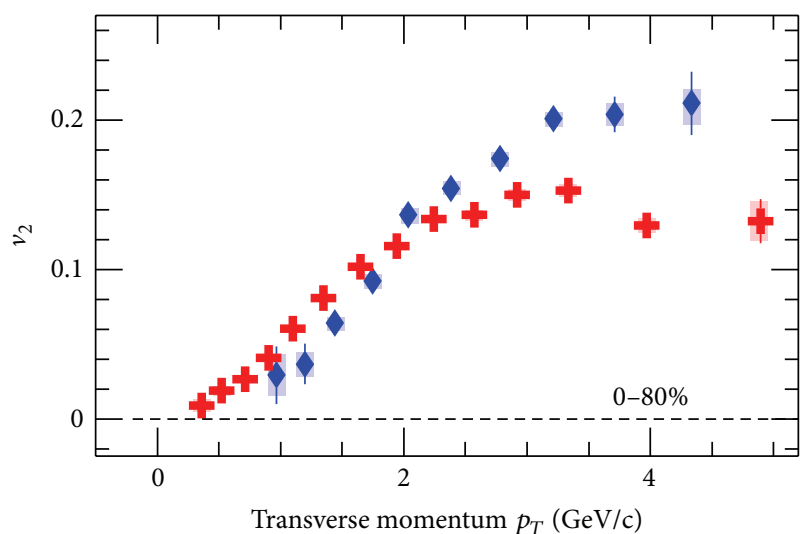

$\Omega$
$\phi$

(a)

(b)

Figure 2: The $v_{2}$ as function of $p_{T}$ for (a) $\pi$ and proton and (b) $\phi, \Omega$ in $\mathrm{Au}+\mathrm{Au}$ collisions at $\sqrt{\boldsymbol{s}_{N N}}=200 \mathrm{GeV}$ for $0-80 \%$ centrality [19].

by number of constituent quarks $\left(n_{q}\right)$ to make $K_{S}^{0}$ and $\Lambda$ results follow the same curve. The results from $0-20 \%$ and $20-60 \%$ central $\mathrm{Cu}+\mathrm{Cu}$ collisions and from $0-10 \%, 10-40 \%$, and $40-80 \%$ central $\mathrm{Au}+\mathrm{Au}$ collisions are presented. For a given collision system, stronger collectivity flow is apparent as higher scaled $v_{2}$ values in more central collisions. For both $\mathrm{Au}$ $+\mathrm{Au}$ and $\mathrm{Cu}+\mathrm{Cu}$ collisions, larger collective flow is observed in larger system size which could be characterized by number of participants. Namely, the collisions with larger number of participants generate larger collective flow.

2.2. Multistrange Hadron and $\phi$ Meson $v_{2}$. STAR experiment presented the first $v_{2}$ results of multistrange hadrons based on $2 \times 10^{6}$ events collected in the year of 2001-2002 [18]. Significant $v_{2}$ signals of $\Xi$ baryons which are similar to results for $\Lambda$ baryons are observed in $\mathrm{Au}+\mathrm{Au}$ collisions at $\sqrt{s_{N N}}=$ $200 \mathrm{GeV}$. At low $p_{T}(<2 \mathrm{GeV} / \mathrm{c})$, the mass ordering is observed for $\Xi v_{2}$ which is in agreement with the hydrodynamic model calculations. Due to limited statistics, the $v_{2}$ of $\Omega$ baryons have large statistical uncertainties, and it is not clear whether $\Omega v_{2}$ follows baryon or meson band at the intermediate $p_{T}$ range $(2-5 \mathrm{GeV} / \mathrm{c})$. But nonzero value of $v_{2}$ was clearly observed at that time. These results suggest that collective motion has been developed at parton phase in $\mathrm{Au}+\mathrm{Au}$ collisions at $\sqrt{s_{N N}}=200 \mathrm{GeV}$.

Later, in the RHIC runs of the year 2010-2011, about 730 million minimum bias events were recorded by STAR. Sufficient statistics of multistrange hadrons and $\phi$ mesons support the precise measurements on $v_{2}$. The multistrange hadrons and the $\phi$ meson were reconstructed despite the following decay channels: $\phi \rightarrow K^{+}+K^{-}, \Xi^{-} \rightarrow \Lambda+\pi^{-}\left(\bar{\Xi}^{+} \rightarrow\right.$ $\left.\bar{\Lambda}+\pi^{+}\right)$, and $\Omega^{-} \rightarrow \Lambda+K^{-}\left(\bar{\Omega}^{+} \rightarrow \bar{\Lambda}+K^{+}\right)$. Figure 2 shows the $v_{2}$ as a function of $p_{T}$ for (a) $\pi$ and protons and (b) $\phi$, $\Omega$ in $\mathrm{Au}+\mathrm{Au}$ collisions at $\sqrt{s_{N N}}=200 \mathrm{GeV}$ for $0-80 \%$ centrality [19]. A comparison between $v_{2}$ of $\pi$ and protons, consisting of up $(u)$ and down $(d)$ light constituent quarks, is shown in panel (a). Correspondingly, panel (b) shows a comparison of $v_{2}$ of $\phi$ and $\Omega$ containing $s$ constituent quarks. This is the first time that high precision measurement of $\Omega$ baryon $v_{2}$ up to $4.5 \mathrm{GeV} / \mathrm{c}$ is available in experiments of heavy ion collisions. In the low $p_{T}$ region $\left(p_{T}<2.0 \mathrm{GeV} / \mathrm{c}\right)$, the $v_{2}$ of $\phi$ and $\Omega$ follows mass ordering. At intermediate $p_{T}\left(2.0<p_{T}<5.0 \mathrm{GeV} / \mathrm{c}\right)$, a baryon-meson separation is observed. The $v_{2}$ results of $\phi$ mesons are consistent in two independent measurements at RHIC, PHENIX [20], and STAR. It is evident that the $v_{2}\left(p_{T}\right)$ of hadrons consisting only of strange constituent quarks ( $\phi$ and $\Omega$ ) is similar to that of light hadrons, $\pi$ and protons. However the $\phi$ and $\Omega$ do not participate strongly in the hadronic interactions, because of the smaller hadronic cross sections compared to $\pi$ and protons. It suggests the major part of the collectivity is developed during the partonic phase in high energy heavy ion collisions. ALICE experiment recently published multistrange hadron and $\phi$ meson $v_{2}$ measurements in $\mathrm{Pb}+\mathrm{Pb}$ collisions at $\sqrt{s_{N N}}=2.76 \mathrm{TeV}$ [21]. Also significant $v_{2}$ values for these particles are observed. Experimental measurements at RHIC and LHC indicate partonic collectivity has been built up in high energy heavy ion collisions.

2.3. Comparison of $\phi$ Meson and Proton $v_{2}$. The $\phi$ meson and proton show different sensitivity on the hadronic rescatterings. As discussed previously, the $\phi$ meson is less sensitive to the late hadron-hadron interactions than light hadrons due to the smaller hadronic cross section. It means light hadrons (e.g., protons) would gain larger additional radial flow which modifies the $v_{2}\left(p_{T}\right)$ shape during final hadronic rescatterings. Hydrodynamical model calculations predict that $v_{2}$ as a function of $p_{T}$ for different particle species follows mass ordering, where the $v_{2}$ of heavier hadrons is lower than that of lighter hadrons [22]. The identified hadron $v_{2}$ measured in experiment indeed proves the mass ordering in the low $p_{T}$ region $\left(p_{T}<2.0 \mathrm{GeV} / \mathrm{c}\right)$. Hirano et al. predict that the mass ordering of $v_{2}$ could be broken between $\phi$ mesons and protons at low $p_{T}\left(p_{T}<1.5 \mathrm{GeV} / \mathrm{c}\right)$ based on a 

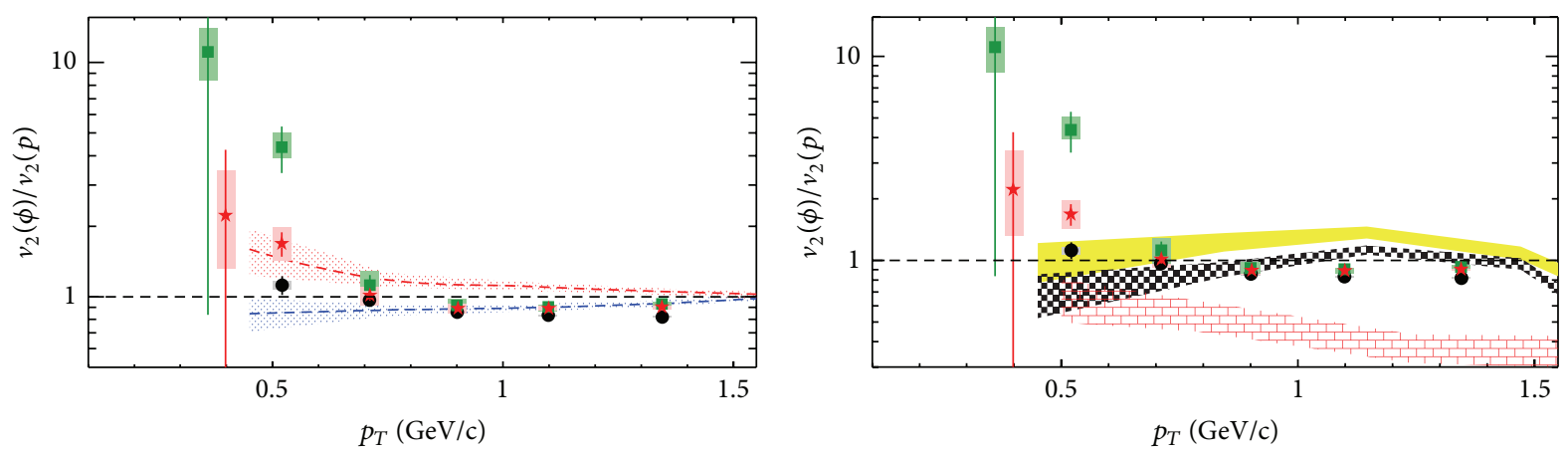

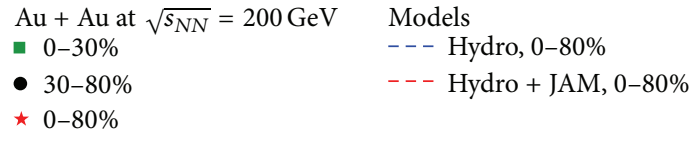

(a)

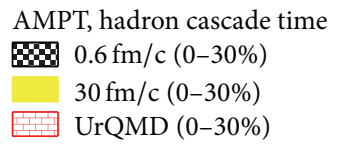

(b)

Figure 3: The ratio of $v_{2}(\phi)$ to $v_{2}(p)$ as function of $p_{T}$ in $\mathrm{Au}+\mathrm{Au}$ collisions at $\sqrt{s_{N N}}=200 \mathrm{GeV}$ for two centralities, $0-30 \%$ and $30-80 \%$. The bands in panels (a) and (b) represent the hydro and transport model calculations for $v_{2}(\phi) / v_{2}(p)$, respectively [19].

model with ideal hydrodynamics plus hadron cascade process $[10,11]$. Here $\phi$ mesons and protons are chosen for the study, as their rest masses are quite close to each other. As the model calculations assign a smaller hadronic cross section for $\phi$ mesons compared to protons, the broken mass ordering is regarded as the different hadronic rescattering contributions on the $\phi$ meson and proton $v_{2}$.

Figure 3 shows the ratios of $\phi v_{2}$ to proton $v_{2}$ from model calculations and experimental data [19]. This ratio is larger than unity at $p_{T} \sim 0.5 \mathrm{GeV} / \mathrm{c}$ for $0-30 \%$ centrality. It indicates breakdown of the expected mass ordering in that momentum range. This could be due to a large effect of hadronic rescatterings on the proton $v_{2}$. The data of $0-80 \%$ centrality around $0.5 \mathrm{GeV} / \mathrm{c}$ quantitatively agrees with hydro + hadron cascade calculations indicated by the shaded red band in panel (a) of Figure 3, even though there is a deviation in higher $p_{T}$ bins. A centrality dependence of $v_{2}(\phi)$ to $v_{2}(p)$ ratio is observed in the experimental data. Namely, the breakdown of mass ordering of $v_{2}$ is more pronounced in $0-30 \%$ central collisions than in 30-80\% peripheral collisions. In the central events, both hadronic and partonic interactions are stronger than in peripheral events. Therefore, the larger effect of late stage hadronic interactions relative to the partonic collectivity produces a greater breakdown of mass ordering in the 0$30 \%$ centrality data than in the $30-80 \%$. This observation indirectly supports the idea that the $\phi$ meson has a smaller hadronic interaction cross section. The ratio of $\phi v_{2}$ to proton $v_{2}$ was also studied by using the transport models AMPT [23] and UrQMD [24]. Panel (b) of Figure 3 shows the $v_{2}(\phi)$ to $v_{2}(p)$ ratio for $0-30 \%$ centrality from AMPT and UrQMD models. The black shaded band is from AMPT with a hadronic cascade time of $0.6 \mathrm{fm} / \mathrm{c}$ while the yellow band is for a hadronic cascade time of $30 \mathrm{fm} / \mathrm{c}$. Larger hadronic cascade time is equivalent to stronger hadronic interactions. It is clear that the $v_{2}(\phi) / v_{2}(p)$ ratio increases with increasing hadronic cascade time. This is attributed to a decrease in the proton $v_{2}$ due to an increase in hadronic rescattering while the $\phi$ meson $v_{2}$ is less affected. The ratios from the UrQMD model are much smaller than unity (shown as a brown shaded band in panel (b) of Figure 3). The UrQMD model lacks partonic collectivity; thus the $\phi$ meson $v_{2}$ is not fully developed. None of these models could describe the detailed shape of the $p_{T}$ dependence. In $\sqrt{s_{N N}}=2.76 \mathrm{TeV} \mathrm{Pb}$ $+\mathrm{Pb}$ collisions at LHC, there is an indication that the $\phi$ meson $v_{2}$ is larger than the proton $v_{2}$ for the lowest $p_{T}$ bin $[21,25]$. Unfortunately, currently the uncertainties on the ALICE $\phi$ meson $v_{2}$ measurements are too large to conclude.

2.4. Number-of-Constituent-Quark Scaling. The number-ofconstituent-quark (NCQ) scaling in $v_{2}$ in the intermediate $p_{T}$ range $\left(2<p_{T}<5 \mathrm{GeV} / \mathrm{c}\right)$ could be well reproduced by the quark coalescence [26] or recombination [27] mechanisms in particle production. The NCQ scaling indicates that the collectivity in the parton level has been achieved in high energy heavy ion collisions at RHIC. Figure 4 shows number of constituent quarks $\left(n_{q}\right)$ scaled $v_{2}$ as a function of transverse momentum scaled by $n_{q}\left(p_{T} / n_{q}\right)$ and transverse mass minus rest mass scaled by $n_{q}\left(\left(m_{T}-m_{0}\right) / n_{q}\right)$ for identified hadrons from $\mathrm{Au}+\mathrm{Au}$ collisions at $\sqrt{s_{N N}}=200 \mathrm{GeV}$ for two centralities, $0-30 \%$ and $30-80 \%$. To investigate the possible system size dependence deviation from NCQ scaling, $K_{S}^{0} v_{2}$ was fitted with a third-order polynomial function. Then the ratio to the $K_{S}^{0}$ fit was calculated. Figures $4(\mathrm{e})-4(\mathrm{~h})$ show the results. Excluding pions, the scaling holds approximately within $10 \%$ for both $0-30 \%$ and $30-80 \%$ centralities. The pion is excluded as it is strongly affected by resonance decay process and nonflow correlations [28, 29]. Figure 5 shows NCQ scaling at LHC energy. The maximum deviation from NCQ scaling is $\sim 20 \%$ at $\sqrt{s_{N N}}=2.76 \mathrm{TeV}$ as observed by ALICE experiment [21]. Therefore, at top RHIC energy, NCQ scaling holds better than LHC energy.

Recently, CMS collaboration presented the $v_{2}$ results of strange hadrons $\left(K_{S}^{0}\right.$ and $\left.\Lambda\right)$ in $p+\mathrm{Pb}$ collisions at $\sqrt{s_{N N}}=$ $5.02 \mathrm{TeV}$ with event sample of large multiplicity [30, 31]. Nice 


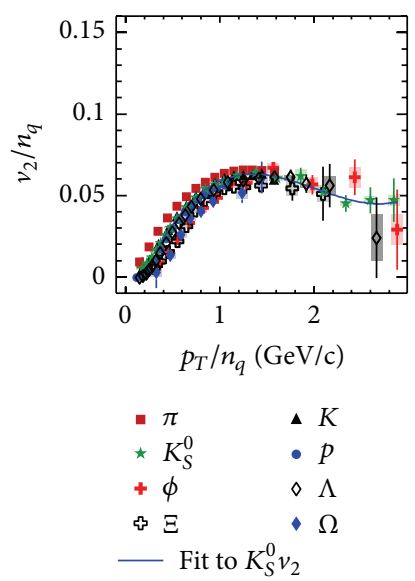

(a) $0-30 \%$

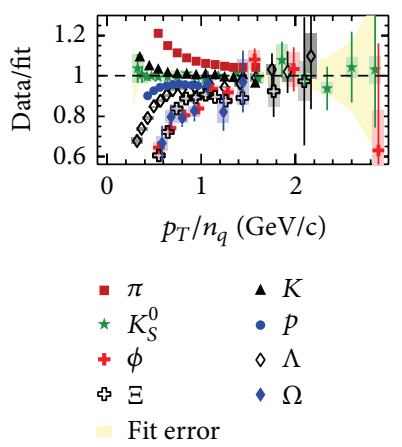

(e) $0-30 \%$

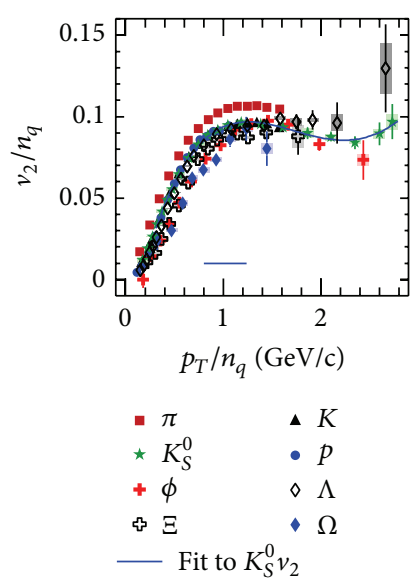

(b) $30-80 \%$

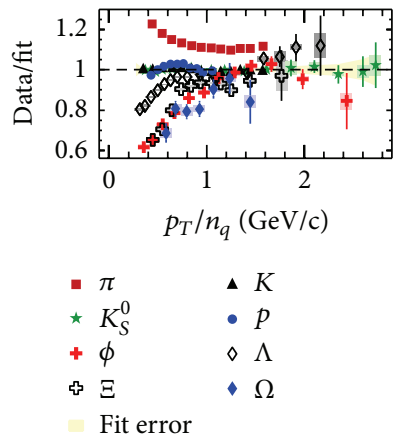

(f) $30-80 \%$

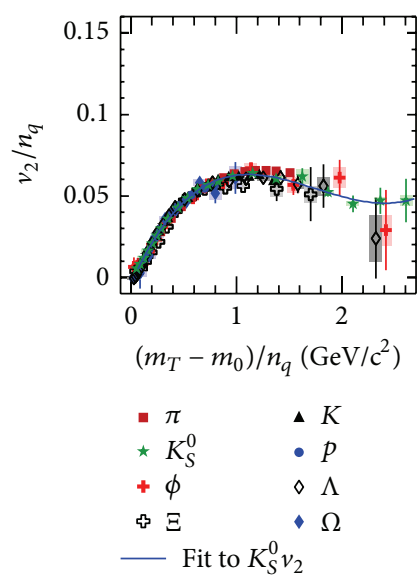

(c) $0-30 \%$

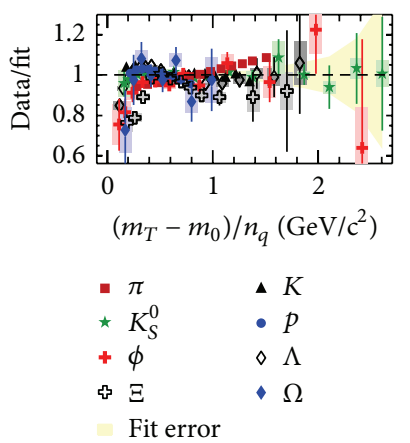

(g) $0-30 \%$

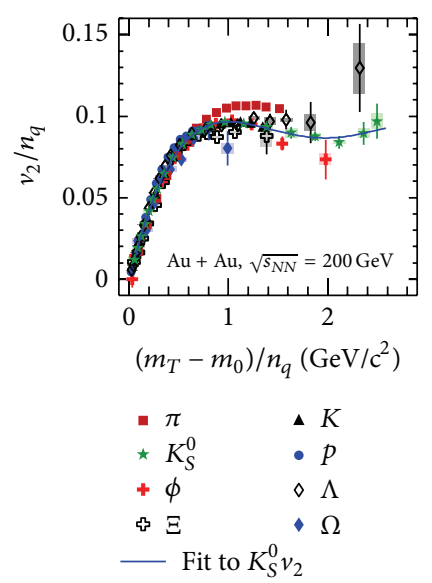

(d) $30-80 \%$

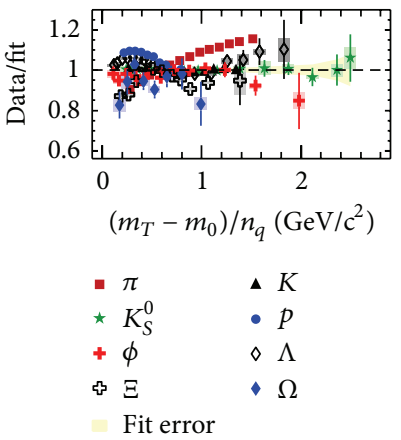

(h) $30-80 \%$

Figure 4: $v_{2}$ scaled by number of constituent quarks $\left(n_{q}\right)$ as a function of $p_{T} / n_{q}$ and $\left(m_{T}-m_{0}\right) / n_{q}$ for identified hadrons from Au + Au collisions at $\sqrt{s_{N N}}=200 \mathrm{GeV}$ for two centralities, $0-30 \%$ and $30-80 \%$. Ratios with respect to a polynomial fit to $K_{S}^{0} v_{2}$ are shown in the corresponding panels (e-h) [19].

NCQ scaling (less than $10 \%$ violation) is observed. It indicates that the partonic level collectivity has been built up even in small $p+\mathrm{Pb}$ colliding system. It would be interesting to compare the NCQ scaling using event samples with large and small multiplicity in the future.

2.5. Beam Energy Dependence. STAR experiment has covered the beam energies of $\sqrt{s_{N N}}=7.7,11.5,14.5,19.6,27,39$, 62.4, and $200 \mathrm{GeV}$. During 2010-2014, a Beam Energy Scan program (phase I) was carried out at RHIC. The main motivation is to explore the nuclear matter phase structure in the higher net-baryon region.

The most striking feature on the $v_{2}$ measurements is the observation of an energy dependent difference in $v_{2}$ between particles and their corresponding antiparticles [32, 33]. Figure 6 shows the difference in $v_{2}$ between particles and their corresponding antiparticles as a function of beam energy. The difference between baryon and antibaryon is much more pronounced than difference between mesons. Proton versus antiproton and $\Lambda$ versus $\bar{\Lambda}$ show the same magnitude of difference. This difference naturally breaks thenumber-of-constituent-quark scaling (NCQ) in $v_{2}$ which is regarded as an evidence of partonic collectivity in the top energy heavy ion collisions at RHIC. It indicates that the hadronic degrees of freedom play a more important role at lower collision energies. The data have also been compared to hydrodynamics + transport (UrQMD) hybrid model [34] and Nambu-Jona-Lasinio (NJL) model [35] which considers both partonic and hadronic potential. The hybrid model could reproduce the baryon (proton) data but fails to explain the mesons, whereas the NJL model could qualitatively reproduce the hadron splitting. However, even if one tunes the $R_{v}$ parameter which is related to the partonic potential, NJL model fails to reproduce the magnitude for all hadron species simultaneously. Analytical hydrodynamic solution can reproduce the data within uncertainties [36]. It predicts $\Delta v_{2}^{p}>\Delta v_{2}^{\Lambda}>\Delta v_{2}^{\Xi}>\Delta v_{2}^{\Omega}$ for baryons. Future high precise data will clarify the validity of this description.

2.6. Comparison with Hydrodynamic Calculations. The $p_{T}$ differential $v_{2}$ could be modified by an increase on both collective and radial flow with increasing of colliding energy. It is qualitatively described by hydrodynamic calculations [37]. The recent comparison between ALICE measurements and model calculations shows a nice agreement in $40-50 \%$ central collisions including strange baryon $\Lambda$ and multistrange baryon $\Xi$. However, for more central collisions (e.g., 

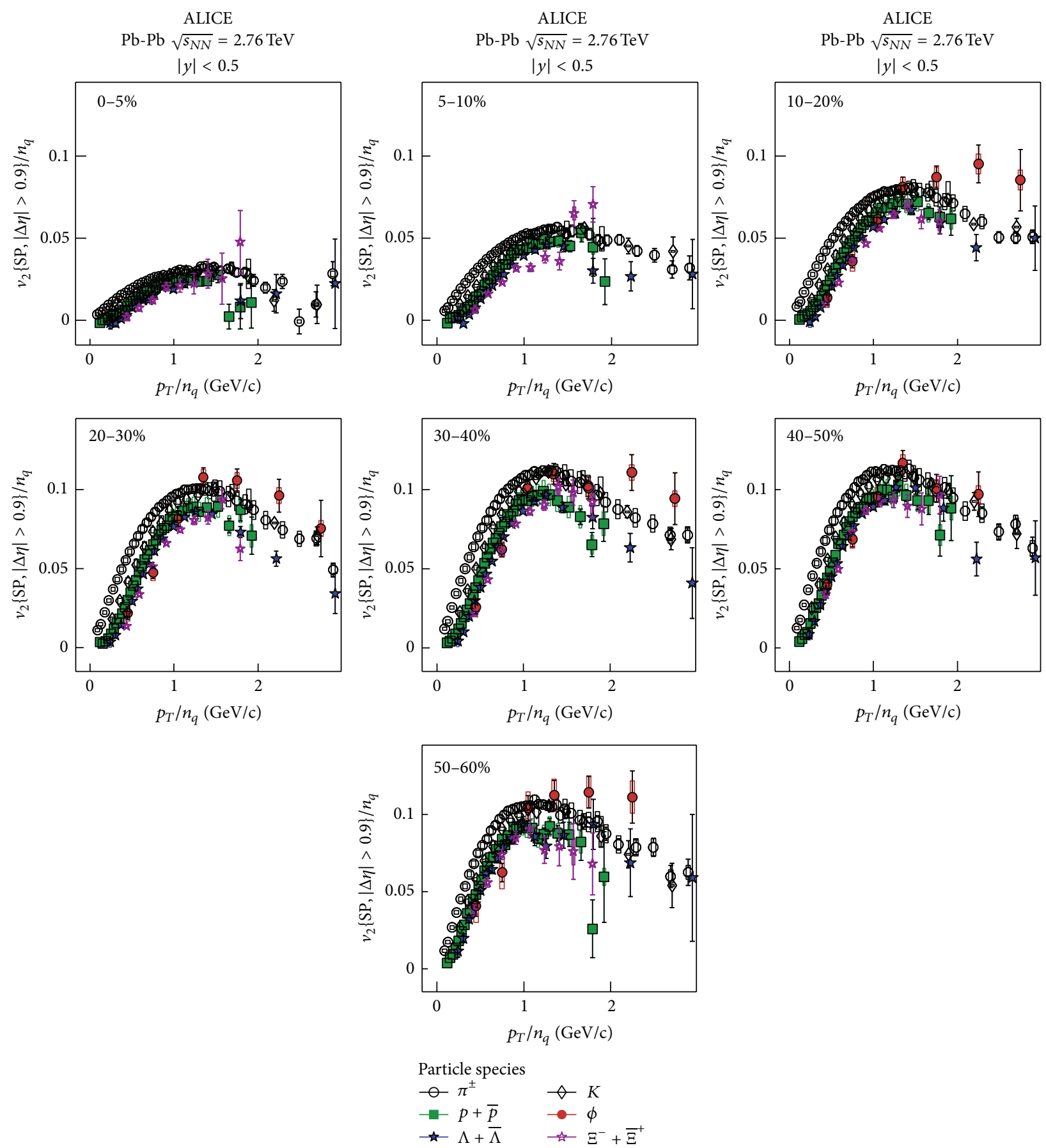

FIGURE 5: $v_{2}$ scaled by number of constituent quarks $\left(n_{q}\right)$ as a function of $p_{T} / n_{q}$ for identified hadrons from $\mathrm{Pb}+\mathrm{Pb}$ collisions at $\sqrt{s_{N N}}=$ $2.76 \mathrm{TeV}$ for various centrality intervals [21].

$10-20 \%)$ a clear discrepancy is observed for protons, $\Lambda$, and $\Xi[21]$.

Later, it was realized that the hadronic rescatterings are important to be included in the hydrodynamic calculations for a fair comparison between data and models [38]. In Figure 7, viscous hydrodynamical calculations with (VISHNU) and without (VISH2+1) a hadronic cascade afterburner are compared. The increase in mass splitting between identified particles for VISHNU (solid curves) compared to VISH2+1 (dashed curves) illustrates the larger radial flow in the VISHNU calculations due to the contribution of the hadronic cascade. The mass splitting between the pions and strange baryons $(\Lambda)$ /multistrange baryons $(\Xi)$ does not change much, as small hadronic rescattering cross sections are assigned to these particles. The mass ordering observed in pure viscous hydrodynamical calculations is not preserved anymore between protons and strange baryons $(\Lambda) /$ multistrange baryons $(\Xi)$ after including the hadronic interactions in 


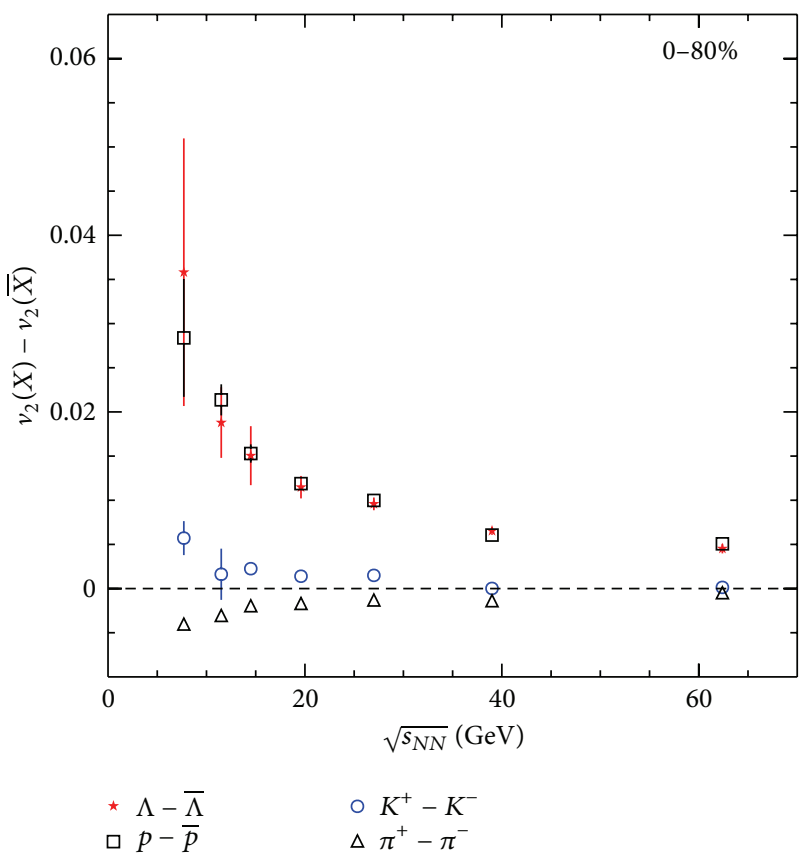

Figure 6: The difference in $v_{2}$ between particles $(X)$ and their corresponding antiparticles $(\bar{X})$ as a function of beam energy for 0$80 \%$ central $\mathrm{Au}+\mathrm{Au}$ collisions $[32,39]$.

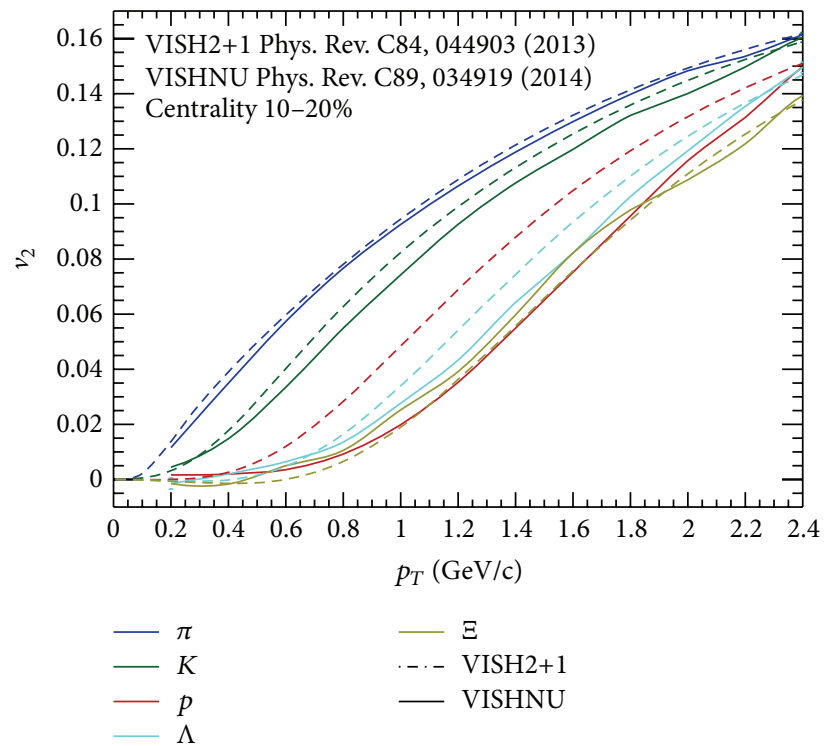

FIgURE 7: The viscous hydrodynamical calculations without a hadronic cascade afterburner (VISH2+1) and with a hadronic cascade afterburner (VISHNU) [25, 37, 38, 40].

VISHNU. Figure 8 shows the comparison between the $p_{T}$ differential $v_{2}$ measured by ALICE and the VISHNU model. Even though VISHNU gives a very well description of kaons, clear discrepancy for protons, $\Lambda$ and $\Xi$, is observed. The VISHNU calculations underpredict the $v_{2}$ of protons and overpredict the $v_{2}$ of $\Lambda$ and $\Xi$. Obviously, the current theoretical framework of viscous hydrodynamics plus a hadron cascade afterburner does not describe the $v_{2}$ as a function of $p_{T}$

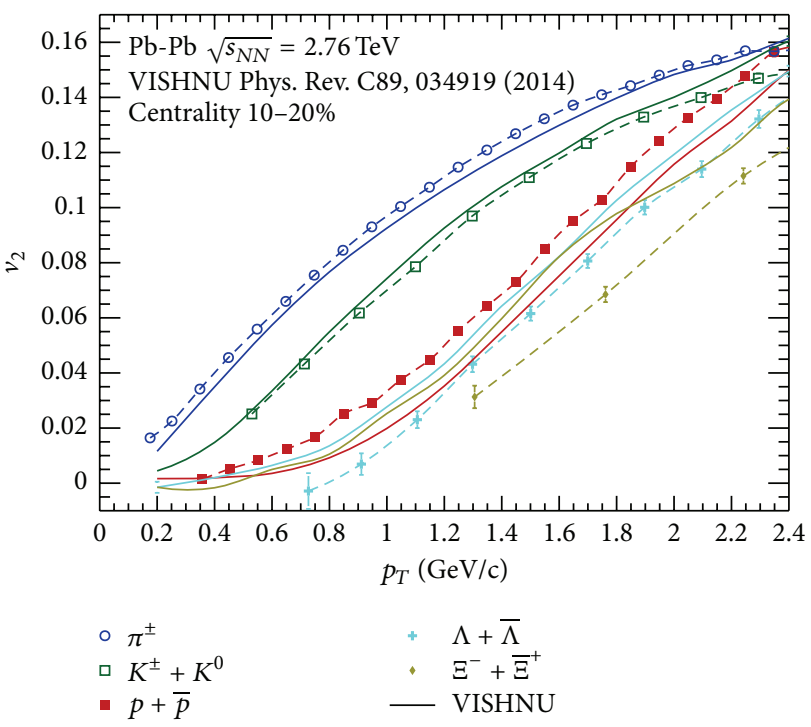

FIGURE 8: The comparison of ALICE measurements and VISHNU model calculations of $v_{2}$ as a function of $p_{T}$ at $\sqrt{s_{N N}}=2.76 \mathrm{TeV}$ for $10-20 \%$ central collisions $[21,25,37,38,40]$.

for identified particles in more central collisions better. One of the possible reasons is that hadronic interaction process for some particle species might not be well understood.

\section{Summary}

In this paper, I review the elliptic flow results of strange and multistrange hadrons in relativistic heavy ion collision from RHIC to LHC energies. The centrality and system size dependence of $v_{2}$ could be described by number of participants in both $\mathrm{Au}+\mathrm{Au}$ and $\mathrm{Cu}+\mathrm{Cu}$ collisions at $\sqrt{s_{N N}}=200 \mathrm{GeV}$. The precise measurements of multistrange hadron $v_{2}$, especially for the $\Omega$ baryons, indicate that the collectivity has been built up in the early partonic stage of collisions. The comparison between the $v_{2}$ of $\phi$ mesons and protons shows a possible violation of hydrodynamics inspired mass ordering in 0-30\% central collisions. It can be qualitatively explained by the different effects of late hadronic interactions on the $\phi$ meson and proton $v_{2}$. The NCQ scaling of identified particles in top energy heavy ion collisions at RHIC is better than LHC energy suggesting that coalescence might be the dominant hadronization mechanism at RHIC in the intermediate transverse momentum region $(2<$ $p_{T}<5 \mathrm{GeV} / \mathrm{c}$ ). Also, the NCQ scaling is observed in small colliding system, $p+\mathrm{Pb}$, at $\sqrt{s_{N N}}=5.02 \mathrm{TeV}$. It indicates that the partonic level of collectivity has also been reached in high energy $p+\mathrm{Pb}$ collisions. At lower beam energy $\left(<\sqrt{s_{N N}}=39 \mathrm{GeV}\right)$, a difference is observed between $v_{2}$ values of particles and antiparticles. Currently there is no theoretical framework that can reproduce the data quantitatively. The recent comparison between viscous hydrodynamic calculations with a hadronic cascade afterburner and experimental data shows a discrepancy on the baryons which challenges the current knowledge on the hadronic interactions. 


\section{Competing Interests}

There is no conflict of interests related to this paper.

\section{Acknowledgments}

This work was supported in part by National Basic Research Program of China (973 program) under Grant no. 2015CB8569, the National Natural Science Foundation of China under Grant no. 11475070, and self-determined research funds of CCNU from the colleges' basic research and operation of MOE under Grant no. CCNU15A02039.

\section{References}

[1] J. Adams, M. M. Aggarwal, Z. Ahammed et al., "Experimental and theoretical challenges in the search for the quark-gluon plasma: the STAR Collaboration's critical assessment of the evidence from RHIC collisions," Nuclear Physics A, vol. 757, no. 1-2, pp. 102-183, 2005.

[2] K. Adcox, S. S. Adler, S. Afanasiev et al., "Formation of dense partonic matter in relativistic nucleus-nucleus collisions at RHIC: experimental evaluation by the PHENIX collaboration," Nuclear Physics A, vol. 757, no. 1-2, pp. 184-283, 2005.

[3] S. Voloshin and Y. Zhang, "Flow study in relativistic nuclear collisions by Fourier expansion of azimuthal particle distributions," Zeitschrift für Physik C: Particles and Fields, vol. 70, no. 4, pp. 665-671, 1996.

[4] A. M. Poskanzer and S. A. Voloshin, "Methods for analyzing anisotropic flow in relativistic nuclear collisions," Physical Review C, vol. 58, no. 3, pp. 1671-1678, 1998.

[5] S. A. Voloshin, A. M. Poskanzer, and R. Snellings, "Collective phenomena in non-central nuclear collisions," https://arxiv.org/ abs/0809.2949.

[6] H. Sorge, "Elliptical flow: a signature for early pressure in ultrarelativistic nucleus-nucleus collisions," Physical Review Letters, vol. 78, no. 12, pp. 2309-2312, 1997.

[7] J.-Y. Ollitrault, "Anisotropy as a signature of transverse collective flow," Physical Review D, vol. 46, no. 1, pp. 229-245, 1992.

[8] H. Sorge, "Soft transverse expansion in $\mathrm{Pb}(158 \mathrm{AGeV})$ on $\mathrm{Pb}$ collisions: preequilibrium motion or first order phase transition?” Physics Letters B, vol. 402, no. 3-4, pp. 251-256, 1997.

[9] D. Teaney, J. Lauret, and E. V. Shuryak, "Flow at the SPS and RHIC as a quark-gluon plasma signature," Physical Review Letters, vol. 86, no. 21, pp. 4783-4786, 2001.

[10] T. Hirano, U. Heinz, D. Kharzeev, R. Lacey, and Y. Nara, "Mass ordering of differential elliptic flow and its violation for $\emptyset$ mesons," Physical Review C, vol. 77, no. 4, Article ID 044909, 2008.

[11] S. Takeuchi, K. Murase, T. Hirano, P. Huovinen, and Y. Nara, "Effects of hadronic rescattering on multistrange hadrons in high-energy nuclear collisions," Physical Review C, vol. 92, no. 4, Article ID 044907, 2015.

[12] A. Shor, " $\varphi$-Meson production as a probe of the quark-gluon plasma," Physical Review Letters, vol. 54, no. 11, pp. 1122-1125, 1985.

[13] H. van Hecke, H. Sorge, and N. Xu, "Evidence of early multistrange hadron freeze-out in high energy nuclear collisions," Physical Review Letters, vol. 81, no. 26, Article ID 5764, 1998.

[14] B. I. Abelev, M. M. Aggarwal, Z. Ahammed et al., "Charged and strange hadron elliptic flow in $\mathrm{Cu}+\mathrm{Cu}$ collisions at
$\sqrt{s_{N N}}=62.4$ and $200 \mathrm{GeV}$,' Physical Review C, vol. 81, no. 4, Article ID 044902, 2010.

[15] B. Alver, B. B. Back, M. D. Baker et al., "High transverse momentum triggered correlations over a large pseudorapidity acceptance in $\mathrm{Au}+$ Au collisions at $\sqrt{s_{N N}}=200 \mathrm{GeV}$," Physical Review Letters, vol. 104, no. 6, Article ID 062301, 2010.

[16] M. L. Miller, K. Reygers, S. J. Sanders, and P. Steinberg, "Glauber modeling in high-energy nuclear collisions," Annual Review of Nuclear and Particle Science, vol. 57, no. 1, pp. 205-243, 2007.

[17] A. Adil, H.-J. Drescher, A. Dumitru, A. Hayashigaki, and Y. Nara, "Eccentricity in heavy-ion collisions from color glass condensate initial conditions," Physical Review C, vol. 74, no. 4, Article ID 044905, 2006.

[18] J. Adams, M. M. Aggarwal, Z. Ahammed et al., "Multistrange Baryon Elliptic Flow in $\mathrm{Au}+\mathrm{Au}$ Collisions at $\sqrt{s_{N N}}=200 \mathrm{GeV}$," Physical Review Letters, vol. 95, no. 12, Article ID 122301, 2005.

[19] L. Adamczyk, J. K. Adkins, G. Agakishiev et al., "Centrality and transverse momentum dependence of elliptic flow of multistrange Hadrons and $\phi$ Meson in $\mathrm{Au}+$ Au collisions at $\sqrt{s_{N N}}=$ 200 GeV," Physical Review Letters, vol. 116, Article ID 062301, 2016.

[20] S. Afanasiev, C. Aidala, N. N. Ajitanand et al., "Elliptic flow for $\phi$ mesons and (anti) deuterons in $\mathrm{Au}+\mathrm{Au}$ collisions at $\sqrt{s_{N N}}=200 \mathrm{GeV}$,' Physical Review Letters, vol. 99, no. 5, Article ID 052301, 2007.

[21] B. B. Abelev, J. Adam, D. Adamová et al., "Elliptic flow of identified hadrons in $\mathrm{Pb}-\mathrm{Pb}$ collisions at $\sqrt{s_{\mathrm{NN}}}=2.76 \mathrm{TeV}$," Journal of High Energy Physics, vol. 2015, no. 6, article 190, 2015.

[22] P. Huovinen, P. F. Kolb, U. Heinz, P. V. Ruuskanen, and S. A. Voloshin, "Radial and elliptic flow at RHIC: further predictions," Physics Letters B, vol. 503, no. 1-2, pp. 58-64, 2001.

[23] Z.-W. Lin, C. M. Ko, B.-A. Li, B. Zhang, and S. Pal, "Multiphase transport model for relativistic heavy ion collisions," Physical Review C, vol. 72, Article ID 064901, 2005.

[24] S. A. Bass, M. Belkacem, M. Bleicher et al., "Microscopic models for ultrarelativistic heavy ion collisions," Progress in Particle and Nuclear Physics, vol. 41, pp. 255-369, 1998.

[25] R. Snellings, J. Aichelin, A. Badalà et al., "How much does the hadronic phase contribute to the observed anisotropic flow at the LHC?” EPJ Web of Conferences, vol. 97, article 25, 2015.

[26] D. Molnár and S. A. Voloshin, "Elliptic flow at large transverse momenta from quark coalescence," Physical Review Letters, vol. 91, no. 9, Article ID 092301, 2003.

[27] R. J. Fries, B. Müller, C. Nonaka, and S. A. Bass, "Hadronization in heavy-ion collisions: recombination and fragmentation of partons," Physical Review Letters, vol. 90, no. 20, Article ID 202303, 4 pages, 2003.

[28] X. Dong, S. Esumi, P. Sorensen, N. Xu, and Z. Xu, "Resonance decay effects on anisotropy parameters," Physics Letters B, vol. 597, no. 3-4, pp. 328-332, 2004.

[29] R. C. Hwa and X. N. Wang, Eds., Quark Gluon Plasma 4, World Scientific, Singapore, 1990.

[30] CMS Collaboration, "Long-range two-particle correlations of strange hadrons with charged particles in $\mathrm{pPb}$ and $\mathrm{PbPb}$ collisions at LHC energies," Physics Letters B, vol. 742, pp. 200224, 2015.

[31] Z. Tu and CMS Collaboration, "Flow of strange and charged particles in $\mathrm{pPb}$ and $\mathrm{PbPb}$ collisions at LHC energies," Journal of Physics: Conference Series, vol. 668, no. 1, Article ID 012030, 2016. 
[32] L. Adamczyk, J. K. Adkins, G. Agakishiev et al., "Elliptic flow of identified hadrons in $\mathrm{Au}+\mathrm{Au}$ collisions at $\sqrt{s_{\mathrm{NN}}}=7.7-$ 62.4 GeV," Physical Review C, vol. 88, no. 1, Article ID 014902, 25 pages, 2013.

[33] L. Adamczyk, G. G. Agakishiev, M. M. Aggarwal et al., "Inclusive charged hadron elliptic flow in $\mathrm{Au}+\mathrm{Au}$ collisions at $\sqrt{s_{N N}}=7.7-39 \mathrm{GeV}$," Physical Review C, vol. 86, Article ID 054908, 2012.

[34] J. Steinheimer, V. Koch, and M. Bleicher, "Hydrodynamics at large baryon densities: understanding proton versus antiproton $v_{2}$ and other puzzles," Physical Review C, vol. 86, no. 4, Article ID 044903, 2013.

[35] J. Xu, T. Song, C. M. Ko, and F. Li, "Elliptic flow splitting as a probe of the QCD phase structure at finite baryon chemical potential," Physical Review Letters, vol. 112, Article ID 012301, 2014.

[36] Y. Hatta, A. Monnai, and B.-W. Xiao, "Flow harmonics $v_{n}$ at finite density," Physical Review D, vol. 92, no. 11, Article ID 114010, 17 pages, 2015.

[37] H. Song, S. A. Bass, and U. Heinz, "Viscous QCD matter in a hybrid hydrodynamic+Boltzmann approach," Physical Review C, vol. 83, no. 2, Article ID 024912, 2011.

[38] H. Song, S. Bass, and U. Heinz, "Spectra and elliptic flow for identified hadrons in $2.76 \mathrm{~A} \mathrm{TeV} \mathrm{Pb}+\mathrm{Pb}$ collisions," Physical Review C, vol. 89, Article ID 034919, 2014.

[39] L. Adamczyk, J. K. Adkins, G. Agakishiev et al., "Centrality dependence of identified particle elliptic flow in relativistic heavy ion collisions at $\sqrt{s_{N N}}=7.7-62.4 \mathrm{GeV}$," Physical Review C, vol. 93, no. 1, Article ID 014907, 2016.

[40] X. Zhu, F. Meng, H. Song, and Y.-X. Liu, "Hybrid model approach for strange and multistrange hadrons in $2.76 \mathrm{~A} \mathrm{TeV}$ $\mathrm{Pb}+\mathrm{Pb}$ collisions," Physical Review C, vol. 91, no. 3, Article ID 034904, 2015. 

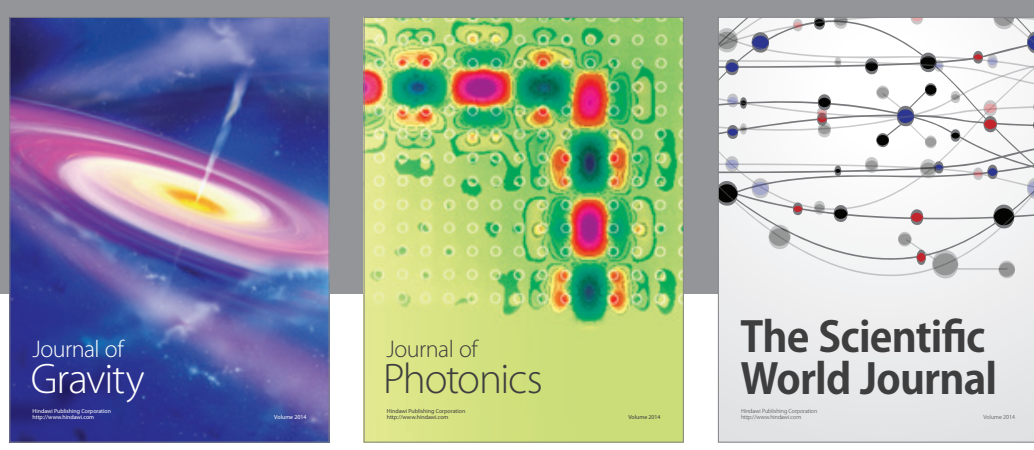

The Scientific World Journal
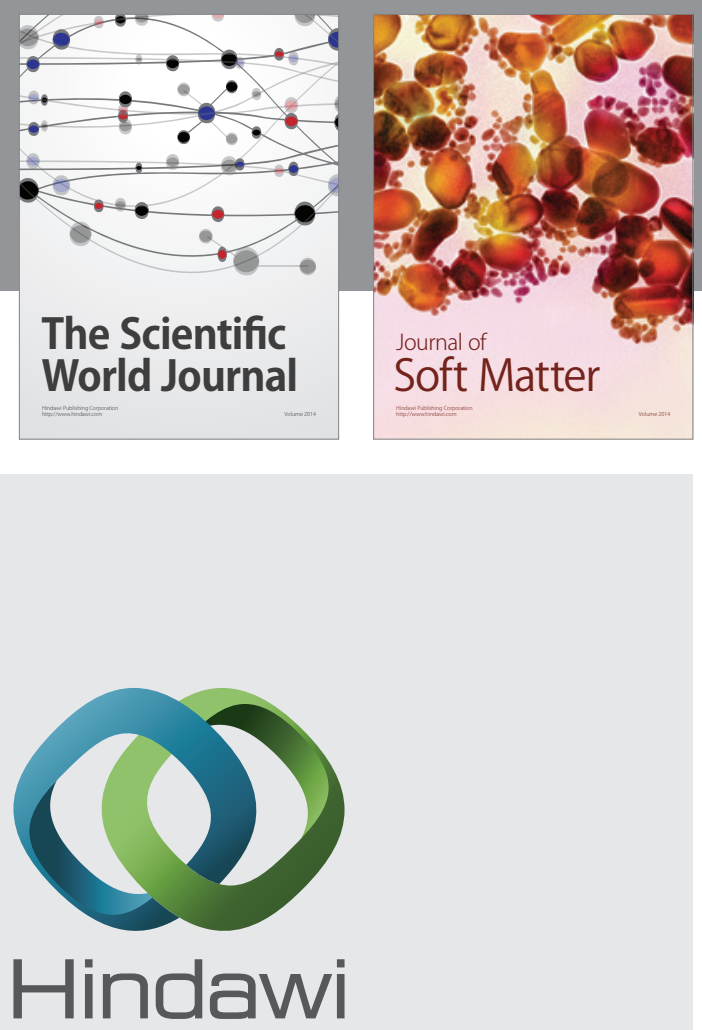

Submit your manuscripts at

http://www.hindawi.com

nternational Journal of

Statistical Mechanics
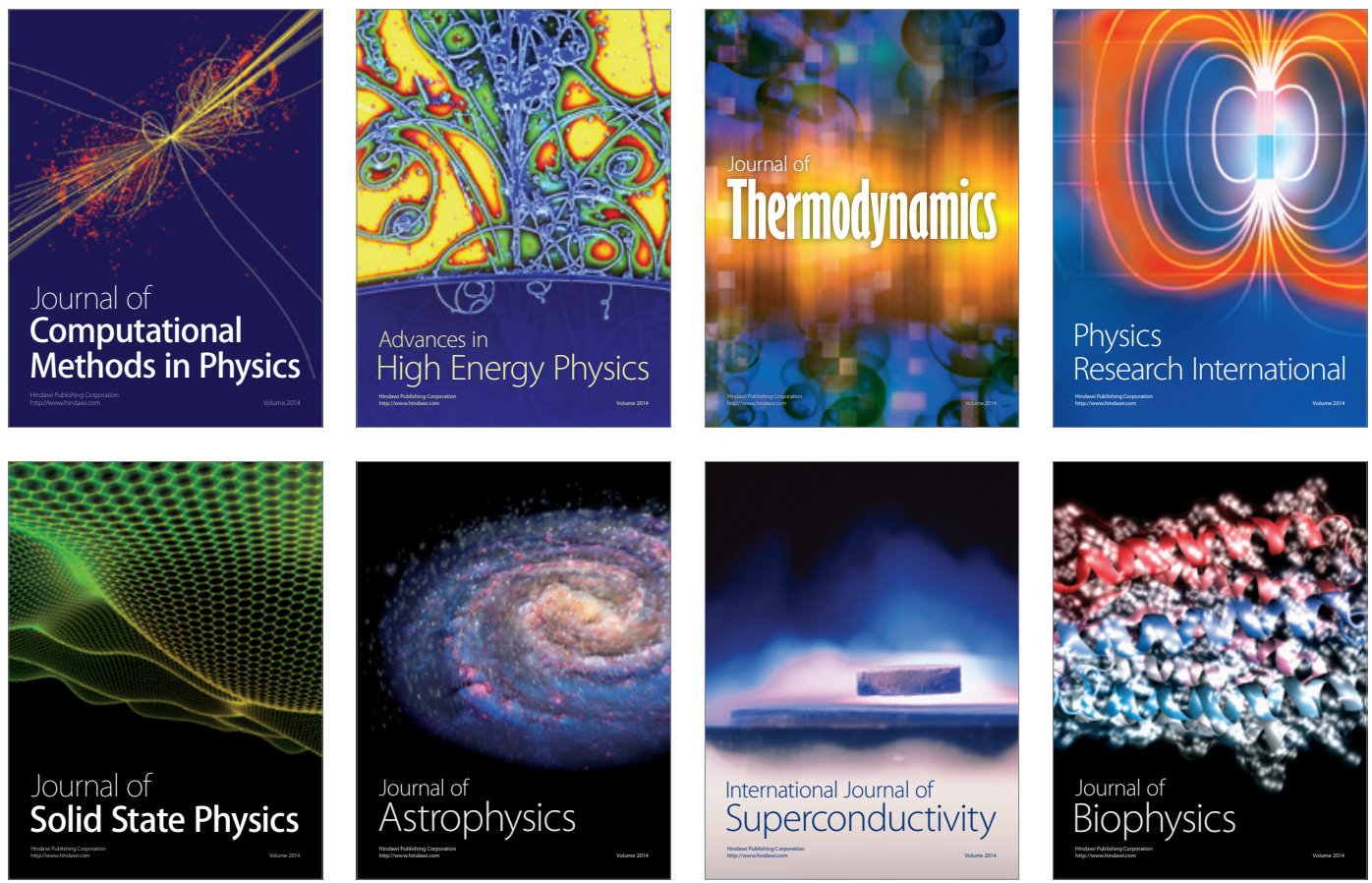
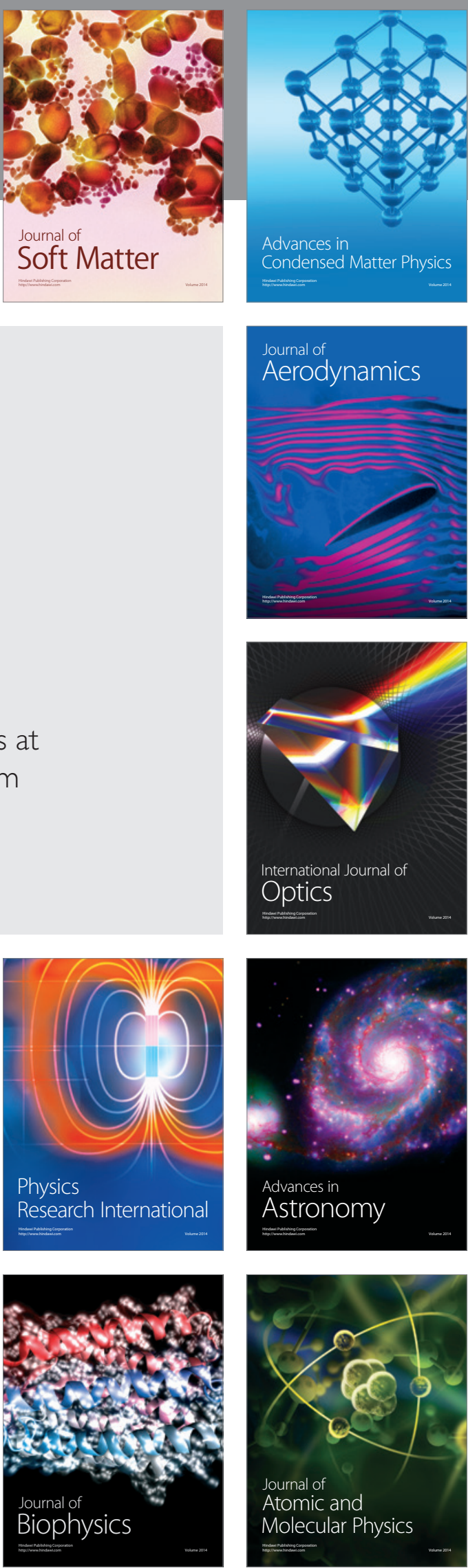